\title{
Use of Dipeptidyl Peptidase-4 Inhibitors in a Subset of Patients with Cystic Fibrosis Related Diabetes
}

\author{
Anery Patel ${ }^{1 *}$, Heather Spain ${ }^{2}$ and Whitney Goldner ${ }^{1}$
}

${ }^{1}$ Department of Internal Medicine, Division of Diabetes, Endocrine and Metabolism, University of Nebraska Medical Center, Omaha, Nebraska, USA ${ }^{2}$ College of Medicine, University of Nebraska Medical Center, Omaha, Nebraska, USA

\begin{abstract}
Cystic Fibrosis Related Diabetes (CFRD) is the most common comorbidity in patients with Cystic Fibrosis (CF) and its incidence is rising. CFRD is associated with a decline in clinical status and six-fold increased mortality in patients with CF, the outcomes of which can be improved with early diagnosis and proper treatment. While current treatment guidelines recommend the use of insulin monotherapy to treat CFRD and do not recommend oral agents, these guidelines were published before the use of dipeptidyl peptidase-4 inhibitors (DPP-4). We present two cases of CFRD patients who were successfully managed with DPP-4 inhibitors both inpatient as well as outpatient.
\end{abstract}

\section{Background}

Cystic fibrosis related diabetes is categorized as "Other forms of Diabetes" by the American Diabetes Association [1] and occurs in nearly $20 \%$ of adolescents and $40-50 \%$ of adults with cystic fibrosis, making it the most common comorbidity of CF [2]. The diagnosis carries with it an associated decrease in pulmonary function, poor nutritional status, and an increased mortality in CF patients [3-6]. Early identification and treatment has been shown to improve survival and measures of overall health, making it vital to successfully diagnose and manage this serious complication to avoid deterioration in clinical status [7].

CFRD typically occurs in patients with severe CF mutations, and is associated with a unique mix of pathophysiologic features that make it unique from type 1 and type 2 diabetes. Although the exact mechanism concerning the development of the disease remains unknown, CFRD is thought to be primarily due to pancreatic insufficiency with patients being primarily insulin insufficient although ketoacidosis is uncommon. However, insulin resistance from chronic infection, parenteral or enteral nutrition and steroid therapy frequently exacerbate hyperglycemia [8].

Current guidelines recommend that CFRD be treated with insulin monotherapy [9]. There is evidence that CF patients who achieve adequate glycemic control on insulin therapy have improvement in weight, protein anabolism, pulmonary function, and survival [9]. Oral agents such as sulfonylureas, metformin, meglitinides, and thiazolidinediones are not as effective as insulin in improving nutritional status and blood glucose control, and subject patients to their negative side effects with limited treatment efficacy so are not recommended for treatment [10-12]. However oral agents can prove to be an appropriate addition in a subset of patients who are reluctant to initiate injectable therapy [8].

Incretins such as Glucagon like peptide (GLP-1) agonists and DPP-4 inhibitors have not been extensively studied in CF patients. There have only been a few studies evaluating the role of incretins in $\mathrm{CF}$. These studies have shown mixed results ranging from normal to impaired GLP-1 and Gastrin Inhibitory Peptide (GIP) secretion after glucola administration during Oral Glucose Tolerance Test (OGTT) in adult as well as pediatric CF patients with and without diabetes mellitus [13-15]. It was also shown that GLP-1 and GIP levels were restored with pancreatic enzyme supplementation [13].
Currently three pilot studies are listed on clinicaltrials.gov to evaluate the role of sitagliptin and its action to increase insulin secretion in CF patients with abnormal glucose tolerance as well as for the prevention of CFRD [16-18]. The results have not yet been published.

Here we describe two interesting cases of use of DPP-4 inhibitors in a subset of CFRD patients as described in detail below.

\section{Case}

\section{Case 1}

38 year old male with CF, CFRD and Gastroesophageal Reflux Disease (GERD) was hospitalized for a CF exacerbation. He was diagnosed with CFRD only weeks before his hospitalization and prior to admit he had no unintentional weight loss or GI symptoms and no history of pancreatitis. He required 2 liters of oxygen at night but did not require chronic steroids or daytime oxygen and was being evaluated for lung transplant. For his CFRD, he had been prescribed pre-meal lispro insulin for blood glucose $>150 \mathrm{mg} / \mathrm{dl}$, however he had not started it prior to admit. His Hemoglobin A1C (A1C) at the time of admission was $7.4 \%$. Vitals were significant for BMI-30 kg/ $/ \mathrm{m}^{2}$ and $\mathrm{O}_{2}$ sat- $95 \%$ on room air. During his hospital stay, he was treated with intravenous (IV) and inhaled antibiotics but did not require steroids. His fasting and premeal blood glucoses were elevated at $180-210 \mathrm{mg} / \mathrm{dl}$, so he was prescribed pre-meal lispro during his hospital stay, but he refused insulin therapy. Hence, we prescribed sitagliptin $100 \mathrm{mg}$ oral daily, after which fasting blood glucoses were in normal range $90-113 \mathrm{mg} / \mathrm{dl}$ and 2 hour postprandial glucose values were consistently less than 180 $\mathrm{mg} / \mathrm{dl}$. He was also able to maintain adequate nutritional intake and

*Corresponding author: Anery Patel, Endocrinology Fellow, Department of Internal Medicine, Division of Diabetes, Endocrinology and Metabolism, 984120 Nebraska Medical Center, Omaha, Nebraska, USA, Tel: 402-559-6876; Fax: 402559-9003; E-mail: anery.patel@gmail.com, anery.patel@unmc.edu

Received October 28, 2014; Accepted February 21, 2015; Published February 25, 2015

Citation: Patel A, Spain H, Goldner W (2015) Use of Dipeptidyl Peptidase-4 Inhibitors in a Subset of Patients with Cystic Fibrosis Related Diabetes. J Diabetes Metab 6: 501. doi:10.4172/2155-6156.1000501

Copyright: (C) 2015 Patel A, et al. This is an open-access article distributed unde the terms of the Creative Commons Attribution License, which permits unrestricted use, distribution, and reproduction in any medium, provided the original author and source are credited. 
weight without GI side effects. He was discharged on sitagliptin $100 \mathrm{mg}$ per day. At a 6 months follow up, A1C improved to 5.8\% and fasting blood glucoses were $100-130 \mathrm{mg} / \mathrm{dl}$ with 2 hour post prandial blood glucose was $80-160 \mathrm{mg} / \mathrm{dl}$. Follow up BMI was $-27 \mathrm{~kg} / \mathrm{m}^{2}$, however, this weight loss was intentional to address his obesity rather than a side effect of the sitagliptin.

\section{Case 2}

53 year old female with CF, CFRD, Crohn's disease, and reactive hypoglycemia was hospitalized for a CF exacerbation. On admission, vitals were significant for BMI $-24 \mathrm{~kg} / \mathrm{m}^{2}$ and $\mathrm{O}_{2}$ sat-98\% on room air. Her CFRD was diagnosed two years prior to this hospitalization. Her predominant symptoms were reactive hypoglycemia rather than fasting or postprandial hyperglycemia, so she had been treated with saxagliptin $5 \mathrm{mg}$ oral daily which eliminated her hypoglycemia and maintained her $\mathrm{A} 1 \mathrm{C}<6 \%$ with premeal blood glucoses of $110-135 \mathrm{mg} /$ dl. She required correction insulin only when receiving steroids as treatment for her CF exacerbations. She had moderate lung disease requiring oral steroids approximately 2-3 times per year. Prior to admit, she had no unintentional weight loss or GI symptoms with no history of pancreatitis. During her hospital stay, she was treated with IV and inhaled antibiotics and did not require steroids. We continued her saxagliptin during hospital stay, all inpatient fasting blood glucose values were $89-111 \mathrm{mg} / \mathrm{dl}$ and pre-meal glucose was $<160 \mathrm{mg} / \mathrm{dl}$. She was discharged on the saxagliptin $5 \mathrm{mg}$ per day. At 6 month follow up, A1C was $6.0 \%$ with fasting blood glucoses of $98-120 \mathrm{mg} / \mathrm{dl}$. She had no weight loss or GI side effects and follow up BMI was stable at $25 \mathrm{~kg} / \mathrm{m}^{2}$.

\section{Discussion}

We describe two patients who responded to DPP-4 inhibitor monotherapy for treatment of early stage CFRD. Both were able to maintain appropriate nutritional status and weight along with adequate blood glucose control without significant side effects.

A combination of factors made them appropriate candidates for DPP-4 inhibitor therapy. Both patients had not been losing weight and had normal or elevated BMI at the time of CFRD diagnosis and at presentation for hospital stay. There was no history of GI symptoms or pancreatitis, or evidence of exocrine insufficiency at baseline. Additionally they were both within two years of CFRD diagnosis and had exacerbations of $\mathrm{CF}$ that did not require steroids or enteral or parenteral nutrition. Admission A1Cs were not significantly elevated but it is known that $\mathrm{A} 1 \mathrm{C}$ can be falsely low in CFRD and cannot be used as reliable indicator of CFRD severity as sole criterion and should be combined with blood glucose measurement [8]. Unfortunately we do not have objective data regarding endogenous insulin production for either patient, including serum insulin or C-peptide levels. However, this would be helpful information to obtain in future prospective studies to determine who may benefit most from this type of therapy. Additionally, studies evaluating the role of DPP-4 inhibitor therapy for beta cell preservation will be important.

The natural history of CFRD is to have progressive insulin deficiency, so DPP-4 inhibitor therapy may not be appropriate for long-term monotherapy, but for early stage CFRD patients with mild CF exacerbations not requiring steroids or enteral or parenteral nutrition. Since potential side effects of DPP-4 inhibitor therapy could be exacerbate GI upset, pancreatitis or potential weight loss, it would not be appropriate for those that are already underweight. It may be most appropriate for those with a normal or elevated BMI, hyperglycemia $<200 \mathrm{mg} / \mathrm{dl}$, and residual endogenous insulin production. There is controversy whether DPP-4 inhibitors cause higher rates of pancreatitis or pancreatic cancer, hence those with a history of pancreatitis or GI symptoms would not be ideal candidates for this treatment [19]. More recent studies show the risk to be minimal but this would need to be considered when starting DPP-4 therapy in this population and would need close monitoring [20].

Current guidelines do not address those patients with CFRD who have normal or increased BMI, early stage of CFRD, minimal insulin requirements and no planned steroids or parenteral or enteral nutrition, or those who will not comply with insulin therapy.

\section{Conclusion}

DPP-4 inhibitors may be a useful alternative therapy for a subset of patients with early stage CFRD. Additional prospective studies need to be conducted to examine the role of this class of medications in CFRD.

\section{References}

1. American Diabetes Association (2014) Standards of Medical Care in Diabetes-2014. Diabetes Care 37: S14-S80.

2. Moran A, Dunitz J, Nathan B, Saeed A, Holme B, et al. (2009) Cystic fibrosisrelated diabetes: current trends in prevalence, incidence, and mortality. Diabetes Care 32: 1626-1631.

3. Milla CE, Billings J, Moran A (2005) Diabetes is associated with dramatically decreased survival in female but not male subjects with cystic fibrosis. Diabetes Care 28: 2131-2144

4. Bismuth E, Laborde K, Taupin P, Velho G, Ribault V, et al. (2008) Glucose tolerance and insulin secretion, morbidity, and death in patients with cystic fibrosis. J Pediatric 152: 540-545.

5. Koch C, Rainisio M, Madessani U, Harms HK, Hodson ME, et al. (2001) Investigators of the European Epidemiologic Registry of Cystic Fibrosis. Presence of cystic fibrosis-related diabetes mellitus is tightly linked to poor lung function in patients with cystic fibrosis; data from the European Epidemiologic Registry of Cystic Fibrosis. Pediatric Pulmonology 32: 343-350.

6. White H, Pollard K, Etherington C, Clifton I, Morton AM, et al. (2009) Nutritional decline in cystic fibrosis-related diabetes; the effect of intensive nutritional intervention. J cyst Fibrosis 8: 179-185.

7. Ode KL, Frohnert B, Laguna T, Phillips J, Holme B, et al. (2010) Oral glucose tolerance testing in children with cystic fibrosis. Pediatric Diabetes 11: 487-492.

8. Fischman D, Nookala VK (2008) Cystic fibrosis-related diabetes mellitus: etiology, evaluation, and management. Endocr Pract 14: 1169-1179.

9. Moran A, Brunzell C, Cohen R, Katz M, Marshall BC, et al. (2010) Clinical care guidelines for cystic fibrosis related diabetes. Diabetes Care 33: 2697-2708.

10. Onady GM, Langdon LJ (2006) Insulin versus oral agents in the management of Cystic Fibrosis Related Diabetes: a case based study. BMC Endocr Disorder $6: 4$

11. Moran A, Pekow P, Grover P, Zorn M, Slovis B, et al. (2009) Cystic Fibrosis Related Diabetes Therapy Study Group. Insulin therapy to improve BMI in cystic fibrosis-related diabetes without fasting hyperglycemia: results of the Cystic Fibrosis Related Diabetes Therapy trial. Diabetes Care 32: 1783-1788.

12. Moran A, Phillips J, Milla C (2001) Insulin and glucose excursion following premeal insulin lispro or repaglinide in cystic fibrosis-related diabetes. Diabetes Care 24: 1706-1710

13. Perano S, Rayner CK, Couper J, Martin J Horowitz M (2014) Cystic fibrosis related diabetes--a new perspective on the optimal management of postprandial glycemia. J Diabetes Complications 28: 904-911.

14. Hillman M, Eriksson L, Mared L, Helgesson K, Landin-Olsson M (2012) Reduced levels of active GLP-1 in patients with cystic fibrosis with and without diabetes mellitus. Journal of cystic fibrosis 11: 144-149.

15. Lanng S, Thorsteinsson B, Roder ME, Orskov C, Holst JJ, et al. (1993) Pancreas and gut hormone responses to oral glucose and intravenous glucagon in cystic fibrosis patients with normal, impaired, and diabetic glucose tolerance. Acta Endocrinol (Copenh) 128: 207-214. 
Citation: Patel A, Spain H, Goldner W (2015) Use of Dipeptidyl Peptidase-4 Inhibitors in a Subset of Patients with Cystic Fibrosis Related Diabetes. J Diabetes Metab 6: 501. doi:10.4172/2155-6156.1000501

Page 3 of 3

16. Stecenko A (2014) Prevention of Cystic Fibrosis Diabetes ClinicalTrials.gov identifier: NCT00967798.

17. Fox L (2014) Treatment of Dysglycemia Using Sitagliptin in Adolescents With Cystic Fibrosis. ClinicalTrials.gov identifier: NCT01721382.

18. University of Pennsylvania (2014) Effect of Chronic Incretin-based Therapy in Cystic FibrosisClinicalTrials.gov identifier: NCT01879228
19. Egan A, Blind E, Dunder K, Graeff P, Hummer BT, et al. (2014) Pancreatic safety of incretin-baed drugs-FDA and EMA assessment. N Engl J Med 370: 794-797.

20. Meier JJ, Nauck MA (2014) Risk of pancreatitis in patients treated with incretinbased therapies. Diabetologia 57: 1320-1324. 